\title{
Tuberculosis and Cerebrovascular Disease: A Review on Pathogenic Mechanisms
}

\author{
Venkatraman $\mathrm{K}$ and Chandramouleeswaran $\mathrm{V}^{*}$ \\ Department of Neurology, Madras Medical College, India
}

Submission: March 18, 2017; Published: October 02,2017

*Corresponding author: Chandramouleeswaran Venkatraman, Professor of Neurology, Institute of Neurology, Madras Medical College and Government General Hospital, Chennai-6000003, Tamilnadu, India, Tel: 919444210890; Email: drvcmnp@gmail.com

\begin{abstract}
The burden of tuberculosis and Cerebrovascular diseases is enormous world-wide. Changing global trends have increasingly made high, middle and low income countries face one of these less exposed burdens. Evidences linking tuberculosis with Cerebrovascular disease have supported the notion of both intraluminal and extraluminal pathology. Vessel wall invasion and inflammation that happens with Tuberculousmeningitis (TBM) has a high level of evidence supporting Tuberculosis as the cause of vasculitis. The basal exudates of TBM have natural course involving the smaller vessels and larger vessels in early and later stages respectively. SPECT along with MR imaging of brain parenchyma and vessels increases the sensitivity and specificity of diagnosis. Steroids play a vital role in reducing the frequency of stroke occurrence and mortality in TBM patients when initiated in early stages. Autoimmune vasculitis secondary to systemic tuberculosis has been demonstrated in Eale's disease affecting the retinal vasculature and Takayasu arteritis affecting the Aorta or its branches. Chronic inflammation in systemic tuberculosis stimulates atherogenesis/plaque rupture in the Tunica intima leading to increased cerebrovascular events. But the level of evidence to this statement is low and therefore warrants further investigation.
\end{abstract}

Keywords: Tuberculosis; Meningitis; Vasculitis; Stroke; Autoimmunity; Takayasu arteritis; inflammation; Atherogenesis

Abbreviations: ANCA: Anti Neutrophil Cytoplasmic Antibody; ATT: Anti Tubercular Therapy; CD: Cluster Determinant; CSF: Cerebrospinal Fluid; CT: Computerised Tomography; ESR: Erythrocyte Sedimentation Rate; HIV: Human Immunodeficiency Virus; HLA: Human Leucocyte Antigen; Hsp: Heat Shock Protein; IL: Interleukin; IFN: Interferon; MPB: Mycobacterial Protein Fraction from BCG; MRI: Magnetic Resonance Imaging; PCR: Polymerised Chain Reaction; PET: Positron Emission Tomography; SPECT : Single Photon Emission Computerised Tomography; TA: Takayasu Arteritis; TB: Tuberculosis; TBM: Tuberculous Meningitis; Th: T helper cell; TNF: Tumour Necrosis Factor; VEGF: Vascular Endothelial Growth Factor; WHO: World Health Organisation

\section{Introduction}

Tuberculosis is a major health hazard to humanity for centuries and is one of the oldest known infections affecting the mankind. In 2015, WHO reported an estimated 10.4 million new cases of Tuberculosis. An estimated 1.4 million HIV negative TB cases died in the same year making the Case fatality rate 17\%. 6 countries of the world namely China, India, Indonesia, Nigeria, South Africa and Pakistan had $60 \%$ of the cases [1]. Unfortunately, the above counties do not lag behind the western world with regards to the burden faced due to stroke. Yearly 15 million people suffer from stoke globally, a third of whom die and another one third left with permanent disability. A larger population below 40 years suffered from stroke in India compared to the Western world.

The association of Tuberculosis with stroke is a major issue in the developing countries where the incidence of TB cases is high. Also the recent issues of immigration have made the western world face this dreaded disease. Tuberculous meningitis (TBM) is a known extra pulmonary site for TB infection which is associated with high incidence (20\% [2] -30\% [3]) of stroke. But does stroke result only from TB meningitis? Does systemic TB infection pose a risk for stroke? This review explores the various mechanisms for stroke occurrence in patients infected with Tuberculosis.

\section{Classification of Vascular Involvement in Tuberculosis}

Vascular involvement culminating in stroke in patients infected with tuberculosis occurs due to the following mechanisms

1. Direct invasion of vessel wall

2. Immunological reaction against the vessel wall

3. Inflammatory mechanisms promoting atherogenesis/ plaque rupture

Among these mechanisms, level of evidence is high for direct vessel wall invasion. Postulated models are available for other mechanisms. 


\section{International Journal of Pulmonary \& Respiratory Sciences}

\section{Direct Invasion of Vessel Wall}

Tuberculous meningitis (TBM) is the prototype entity which results in stroke due to vessel wall infiltration. Meningial deposit of the bacilli follows emboli from tuberculous lesions in lung, kidney and bone. Children are more affected than adults. A dense gelatinous fibrinocellular exudate accumulates in the leptomeninges, particularly in the interpeduncular fossa. The exudate widens two layers of leptomeninges and then spreads anteriorly to encircle anterior cerebral vessels and optic chiasma and laterally into the sylvian fissure to encircle the carotid, middle cerebral vessels and the penetrating branches. Posterior spread marks the pontomesencephalic and medullary cistern involvement with blocking of Foramen of Lushka [4].

\section{Pathogenesis}

The cellular reaction in the meninges is directly proportional to the duration of the illness. A chronic course witnesses lymphocytic and plasma cell infiltration. Following the cellular reaction inflammatory vasculitisoccurs. This is marked by 3 stages.

I. Infiltration

\section{Proliferation}

\section{Necrotizing changes}

The tubercle bacilli encountered by astroglia, endothelial cell and monocyte induce cell adhesion molecule expression and thus neutrophil recruitment throughdiapedesisinto the CSF. Neutrophil secretes vasoactive peptides leading to disruption of blood brain barrier and recruitment of lymphocytes and macrophages through cytokines. Fibrinousexudates containing these inflammatory cells accumulate in the basal meninges. The lymphocytes along with plasma cell infiltrate the vessel wall progressively from Tunica adventitia towards Tunica intima (Figure 1). Among the cytokines, CSF IL- 6 and TNF - alfa have positive correlation with severity and progression of TBM. The infiltration of the intima is followed 2-3 weeks later by proliferation of smooth cells and collagen. This with or without thrombus formation causes vessel wall occlusion and results in infarction (Figure 2). Among the less common mechanisms which result in stroke are the fibrinoid necrosis of vessel wall andvenous sinus thrombosis, implicated in the rare occurrence of hemorrhagic lesion in TBM. Thrombosis in TBM is a rare phenomenon and is due to hypercoagulable state in the acute phase of more severe forms (Stage 3 and 4) of TBM (Table 1) [5].

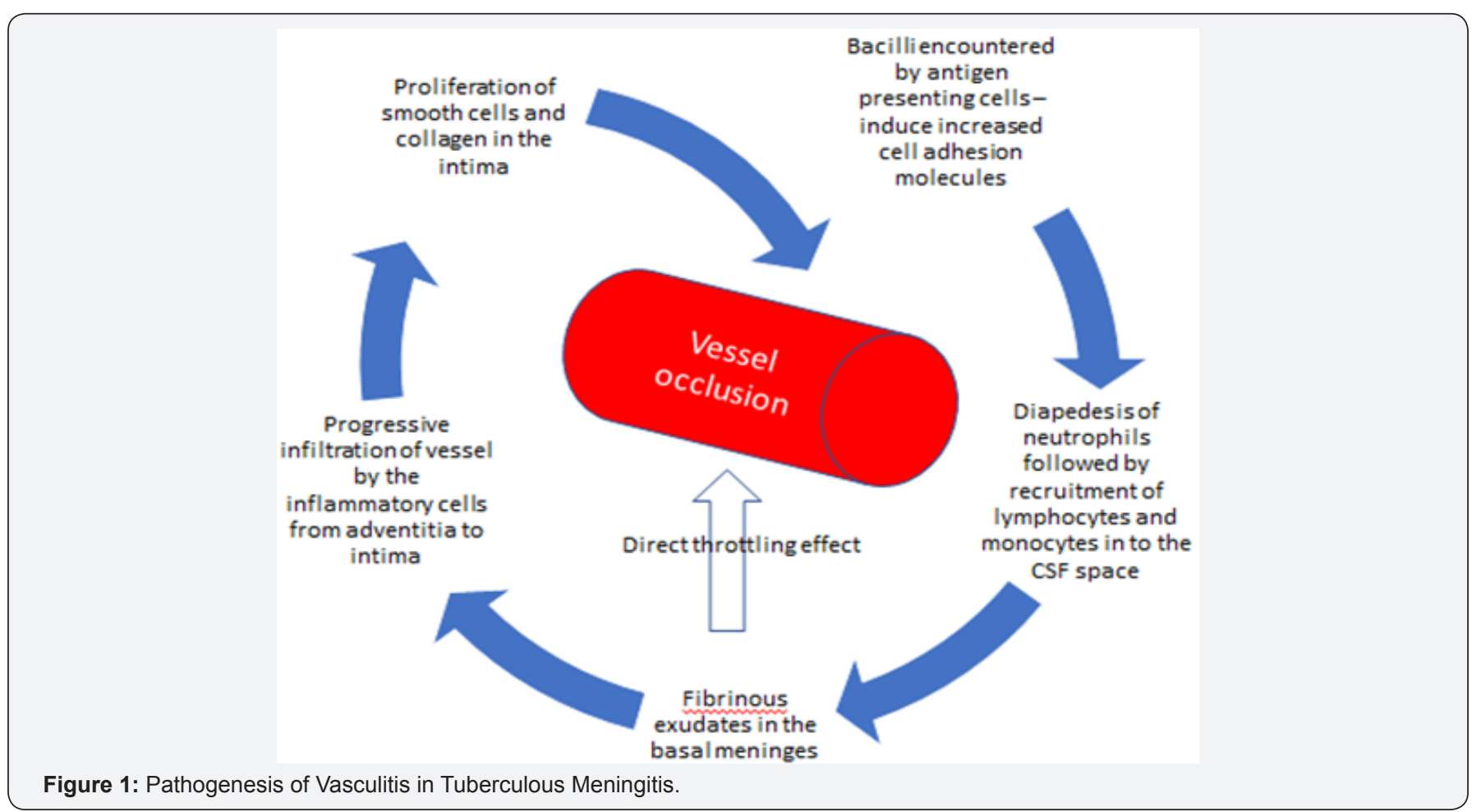

Table 1: Vellore grading of tubercular meningitis with hydrocephalus.

\begin{tabular}{|c|c|}
\hline Grade & Clinical Picture \\
\hline I & Headache, vomiting, fever+/- neck stiffness \\
\hline & No neurological deficits \\
\hline II & Normal sensorium \\
\hline & Neurological deficits \\
\hline III & Normal sensorium \\
\hline & Altered sensorium but easily arousable \\
\hline
\end{tabular}




\section{International Journal of Pulmonary \& Respiratory Sciences}

\begin{tabular}{|c|c|}
\hline & Dense neurological deficit may or may not be present \\
\hline IV & Deeply comatose \\
\hline & Decerebrate or Decorticate posturing \\
\hline
\end{tabular}

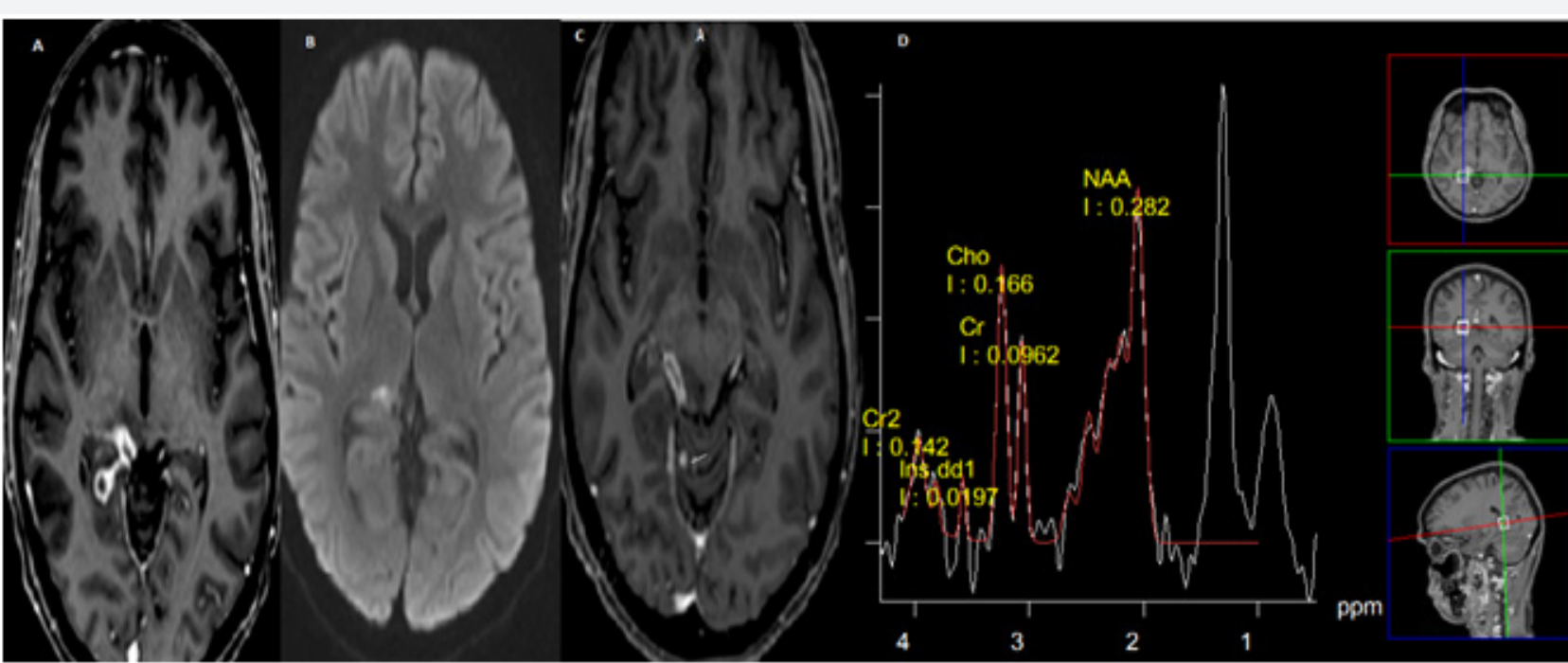

Figure 2: MR imaging of a 35 year old male diagnosed with TBM showing $(A)$ ring enhancing lesion in the right medial temporal cortex (B) acute infarct in the dorsomedial thalami (C) Narrowing of the P2,P3 segments of right PCA artery surrounded by contrast enhancing dense exudates in the perimesencephalic cistern, also noted is a ring enhancing lesion of right cerebellum (D) Magnetic Resonance Spectroscopy showing a Lipid peak(last wave) over the ring enhancing lesion in the temporal lobe suggesting Tuberculosis.

\section{Clinical features}

Patients present with weakness in monoplegic pattern in the acute stage reflecting the small vessel occlusion and then in hemiplegic/quadreplegicpattern, decortication posture in the chronic stages reflecting middle cerebral artery and internal carotid arteryocclusion, thus inferring that longer duration of TBM is associated with higher incidence of stroke. TBM vasculitis is insidious and is not characterized by Transient Ischemic Attacks associated with atherothrombotic strokes.
Also aphasia, agnosia and hemianopia are less uncommon. Cranial neuropathies occur along with motor deficit due to direct compressive effect from the exudates and when present along with infarction increases the possibility of TBM rather than acute bacterial meningitis. Detecting the focal neurological deficits is clinically challenging owing to the altered sensorium in TBM. Stroke in TBM reflects a poor outcome both in terms of morbidity and mortality. Advanced stage of tuberculous meningitis, basal exudates, optochiasmaticarachnoiditis and vision impairment are significant predictors of stroke in patients with TBM [3].

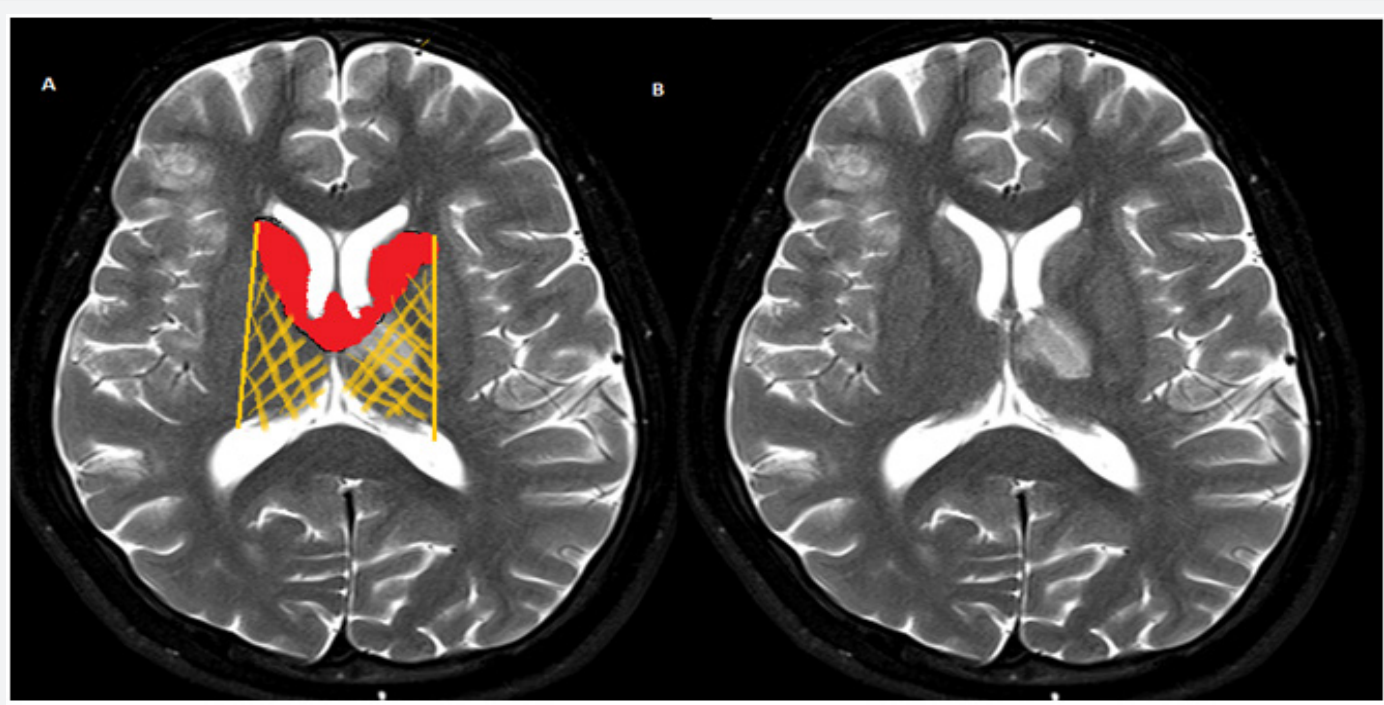

Figure 3: (A)MR image showing the "Tubercular area" or area of infarction (RED)- Caudate, genu of internal capsule, Anterolateral thalam and ischemic zone( ORANGE STRIPES)-posterior limb of internal capsule, lentiform and posterolateral thalami. (B) genu of the internal capsule showing infarct in a 14 year old girl affected with TBM. 


\section{Neuroimaging}

MRI is sensitive than CT in detecting the infarcts in patients with focal neurological defects and also in patients with altered sensorium with a high suspicion of vascular pathology. TBM vasculitis affects specific vessels and specific areas of brain. Basal ganglionic infarctions comprising the head of the caudate, anteromedial thalami, anterior limb and genu of internal capsule are the commonest affected regions of the brain and are commonly referred to as the "Tubercular Zone" (Figure 3). Small vessels namely medial striate, thalamotuberal and thalamoperforate arteries are implicated.

The classical TBM triad in cerebral angiogram includes the 1) narrowing of supraclinoid portion of internal carotid artery 2) widely sweeping pericallosal artery or outward bowing of thalamostriatevein, and 3) delayed circulation in middle cerebral artery with scantly collaterals and early draining veins suggesting a dilated ventricular system and constricted carotid and middle cerebral vessels due to arteritis. Such a triad cannot be appreciated in all the patients and a larger population (57\%) showed normal angiographic studies.

Collaterals arise around the arteries affected by TBM and have been classified into three forms.

a. net like clusters of thin vessels in the region of basal ganglia and base of the brain which resemble Moyamoya like pattern

b. transdural external-internal carotid anastomosis

c. intracranial cortical anastomosis with altered architectural arrangement

SPECT scan is more sensitive in picking up the hypoperfusion than angiographicstudies. Adding MRimaging helps to differentiate a vasculitic cause from a periventricular edema secondary to a hydrocephalus when the SPECT shows hypoperfusion in the basal ganglia region.

\section{Treatment}

Patients benefit with combined steroid and standard antituberculartherapy (ATT). Beneficial effects of steroid especially intravenous dexamethasone have been demonstrated in various studies. Steroids reduce the frequency of infarction and mortality when started early (Stage 1 TBM), but they do not

Table 2: Staging system of Eale's disease.

\begin{tabular}{|c|c|}
\hline Stage & Description \\
\hline I & Periphlebitis of small (Ia) and large caliber (Ib) vessels with superficial retinal hemorrhages \\
\hline IIa & Capillary nonperfusion \\
\hline IIb & Revascularisation else whwere / of the disc \\
\hline IIIa & Fibrovascular proliferation \\
\hline IIIb & Vitreous hemorrhage \\
\hline IVa & Traction/combined rhegmatogenous retinal detachment \\
\hline IVb & Rubeosisiridis, neovascular glaucoma, complicated cataract and optic atrophy \\
\hline
\end{tabular}

improve the long term disability of the TBM patients affected with hemiplegia prior to starting medications. Also, the mortality rates in later stages of TBM are not affected by steroids.

\section{Immunological Reaction Against Vessel Wall}

Tuberculous infection anywhere in the body can mount an immunological reaction against the vessel wall due to the molecular mimicry between TB bacilli and vessel wall antigens. There are two autoimmune vasculitic conditions in which TB has been strongly proposed as the trigger factor

\section{EALE'S DISEASE}

2. TAKAYASU ARTERITIS

\section{Eale's Disease}

Eale's disease is an obliterative vasculopathy involving the retinal vessels in young males (>97\%) predominantly between 20-30 years of age.

Etiopathogenesis: Multiple models have been proposed linking the inflammatory and autoimmune mechanisms in Eale's disease. Of these the most favoured is the one which links Eale's disease with Tuberculosis. More than $70 \%$ of patients had a positive Mycobacterium species detected by PCR from the epiretinal membrane. Also MPB 64 gene and genome of Mycobacterium tuberculosis were demonstrated in more than $50 \%$ of patients from the epiretinal membrane and vitreous humour respectively. Individuals with predisposition to HLAB5, DR1 and DR5 are more vulnerable. Elevated serum IL-6, VEGF levels and increased monocytes are seen. Non-classical CD $16+$ monocytes with a high expression of Toll like Receptor 2 on the cell surface are observed confirming cell mediated autoimmunity directed against the vessels. The above mechanism leads to inflammation of the peripheral retinal vessels with occlusion and retinal infarcts occur along with neovascularization and vitreous hemorrhage.

Clinical Features: Patients remain asymptomatic early in the course of the disease. They notice floaters and blurring of vision which might worsen to a level of complete blindness. Visual symptoms are bilateral in 50\%-90\% population. A new staging system based on the ocular findings has been proposed (Table 2). Nervous system abnormalities including myelopathy, ischemic stroke, bilateral white matter abnormalities in MR imaging and vestibuloauditory dysfunction have been reported less commonly. 
Treatment: Corticosteroids form the mainstay of treatment. Patients not responding to steroids are switched to anti VEGF monoclonal antibody Bevacizumab. Patients showing strong positive reaction to purified protein derivative are also given a 9 month ATT. Studies support Vitamins and antioxidants. Ophthalmological interventions like photocoagulation and vitrectomy are indicated in proliferative stage and vitreous hemorrhage respectively.

\section{Takyasu Arteritis}

Takayasuarteritis (TA) is a systemic inflammatory granulomatous vasculitis of large and medium vessels affecting the aorta and its branches.

Etipathogenesis: Tuberculosis has been strongly linked to TA in various studies. One study demonstrated IS6110 and Hup B gene sequences of Mycobacterium tuberculosis in more than $70 \%$ of aortic tissues of TA patients. Also Real time - PCR was $93.87 \%$ and $98.69 \%$ sensitive and specific in detected the M. tuberculosis bacilli from the aortic tissues of TA patients. An Indian study estimates a 44.8 times higher chance of developing TA in patients with active tuberculosis compared to healthy population [6,7]. Latent tuberculosis detected by Tuberculin Skin Test and Interferon Gamma release assays is more positive in TA patients than general population. A molecular mimicry occurs between the Heat shock protein (hsp) expressed by humans and M. tuberculosis bacilli. Due to an unknown trigger human expresses the mhsp 65 protein of M. tuberculosis in the aorta. This induces the Histocompatibility complex class I chain A (MIC-A) on vessel wall recognised by the Natural killer cells of innate immunity. These cells release perforin on the vessel wall and induce inflammation by recruiting Th1 and Th 17 Cells. These cells along with their corresponding cytokines induce aortic arteritis [8].

Clinical Features: The commonest presentations are hypertension secondary to renal arterial stenosis and cardiac failure. Neurological involvement consists of headache (50$70 \%$ ), visual disturbances (16-35\%) and strokes (5-9\%) due to involvement of carotid and vertebral arteries. Diagnosis confirmed using both blood tests and imaging. Raised ESR, positive ANCA titres are sensitive tests. MR/CT angiogram showing a focal/ segmental non arteriosclerotic narrowing or occlusion of aorta, its primary branches or large arteries of extremities increases the specificity. Vascular PET is the gold standard investigation when available.

Treatment: Corticosteroids are the mainstay in acute stage followed by immunomodulation with either cytotoxic agents like cyclophosphamide, azathioprines, methotrexate or biologicals like TNF inhibitor-etanercept.

\section{Inflammatory Mechanisms Promoting Atherogenesis/ Plaque Rupture}

Commonest cause of ischemic stroke globally is atherothrombosis and thrombo embolism. Systemic tuberculosis as a risk factor promoting atherogenesis in the cardiovascular and cerebrovascular vessels is an issue of debate. Studies have both argued and refuted this hypothesis and therefore the level of evidence is low. A Taiwan based 3 year follow up study using a large cohort showed that patients affected by tuberculosis and completed treatment had a 1.52 times higher risk of developing a stroke than age matched population, after adjusting for other stroke risk factors [9]. Another Taiwan study refuted the increase occurrence of stroke in non-central nervous system Tuberculosis $[10,11]$. Extrapolating the information from the studies supporting higher cardiovascular events related with systemic tuberculosis, to the cerebrovascular events gives us the mechanism underlying the atherogenesis.

\section{Pathogenesis}

1) Increased expression of pro-inflammatory cytokines (i.e., IL-1, IL-2, IL-6, IFN- $\gamma$, TNF- $\alpha$ )

2) Monocyte/macrophage immune activation

3) CD4+ TH1 and TH17 cell immune activation

4) Auto-immunity mediated by antibodies against mycobacterial HSP65( similar to Takayasu arteritis)

Together these mechanisms cause endothelial activation, increased expression of adhesion molecules in the tunica intima. This attracts the immune cells (lymphocytes, monocytes) and thereby promotes atherosclerosis by formation of foam cells in the sub intimal space. This increases the plaque size in the subintimal space and then recruits the smooth cells to form the cap of the plaque along with collagen. Inflammation also causes rupture of the plaque as a result of Matrix metalloproteinase.

The current evidence is insufficient to relate the atherogenic cerebrovascular events to Tuberculosis and will require further investigations.

\section{Conclusion}

Evidences linking tuberculosis to Cerebraovasular events through systemic vasculitis and atherogenesis are surprising. Models proposed both in the immunological and inflammatory frontiers look explanatory, but are incomplete. Further research is requires to investigate the link between Tuberculosis and Cerebrovascular disease.

\section{References}

1. WHO Global Tuberculosis Report 2015

2. Thomas MD, Chopra JS, Walia BNS (1977) Tuberculous meningitis (TBM): a clinical study of 232 cases. J Assoc Physicians India 25(9): 633-639.

3. Anuradha HK, Garg RK, Agarwal A, Sinha MK, Verma R, et al. (2010) Predictors of stroke in patients of tuberculous meningitisand its effect on the outcome. QJM 103(9): 671-678.

4. Misra UK, Kalita J, Maurya PK (2011) Stroke in tuberculous meningitis. Journal of the Neurological Sciences 303 (1-2): 22-30.

5. Rajshekhar V (2009) Management of hydrocephalus in patients with tubercular meningitis. Neurology India 57(4): 368-374. 
6. Kinare SG (1970) Aortitis in early life in India and its association with tuberculosis. J Pathol 100(1): 69-76.

7. Chogle AR, Jain S, Kushwaha H (2105) Mycobacterium theory regarding pathogenesis of Takayasu's arteritis: Numerous unsolved dilemmas. Internet Journal of Rheumatology and Clinical Immunology 3(1).

8. Sheu JJ, Chiou HY, Kang JH, Chen YH, Lin HC (2010) Tuberculosis and the Risk of Ischemic Stroke A 3-Year Follow-Up Study. Stroke 41(2): 244-249.

9. Wu CH, Chen LS, Yen MF, Chiu YH, Fann CY, et al. (2014) Does NonCentral Nervous System Tuberculosis Increase the Risk of Ischemic
Stroke? A Population-Based Propensity Score-Matched Follow-Up Study. PLoS One 9(7): e98158.

10. Huaman MA, Henson D, Ticona E, Sterling TR, Garvy BA (2015) Tuberculosis and cardiovascular disease: linking the epidemics. Tropical Diseases, Travel Medicine and Vaccines 1: 10 .

11. Biswas J, Ravi RK, Naryanasamy A, Kulandai LT, Madhavan HN (2013) Eales' disease - current concepts in diagnosis and management. J Ophthalmic Inflamm Infect 3(1): 11.

Your next submission with Juniper Publishers will reach you the below assets

- Quality Editorial service

- Swift Peer Review

- Reprints availability

- E-prints Service

- Manuscript Podcast for convenient understanding

- Global attainment for your research

- Manuscript accessibility in different formats ( Pdf, E-pub, Full Text, Audio)

- Unceasing customer service

Track the below URL for one-step submission https://juniperpublishers.com/online-submission.php 